\title{
FFLO Superconductivity near the Antiferromagnetic Quantum Critical Point
}

\author{
Youichi YANASE* \\ Department of Physics, University of Tokyo, Tokyo 113-0033, Japan
}

(Received Today 2008)

\begin{abstract}
The Fulde-Ferrel-Larkin-Ovchinnikov (FFLO) state near the antiferromagnetic quantum critical point $(\mathrm{AFQCP})$ is investigated by analyzing the two dimensional Hubbard model on the basis of the fluctuation exchange (FLEX) approximation. The phase diagram against the magnetic field and temperature is compared with that obtained in the BCS theory. We discuss the influences of the antiferromagnetic spin fluctuation through the quasiparticle scattering, retardation effect, parity mixing and internal magnetic field. It is shown that the FFLO state is stable in the vicinity of AFQCP even though the quasiparticle scattering due to the spin fluctuation is destructive to the FFLO state. The large positive slope $\mathrm{d} H_{\mathrm{FFLO}} / \mathrm{d} T$ and the convex curvature $\left(\mathrm{d}^{2} H_{\mathrm{FFLO}} / \mathrm{d} T^{2}>0\right)$ are obtained, where $H_{\mathrm{FFLO}}$ is the critical magnetic field for the second order phase transition from the uniform BCS state to the FFLO state. These results are consistent with the experimental results in $\mathrm{CeCoIn}_{5}$. The possible magnetic transition in the FFLO state is examined.
\end{abstract}

KEYWORDS: FFLO superconductivity; antiferromagnetic quantum critical point

The BCS theory assumes the Cooper pairs which have zero total momentum. ${ }^{1}$ The Cooper pairing with finite total momentum was predicted in 1960's by Fulde and Ferrel $^{2}$ and Larkin and Ovchinnikov. ${ }^{3}$ The FFLO state can be stabilized in the presence of the high magnetic field or the molecular field. In addition to the U(1)-gauge symmetry the spatial symmetry is spontaneously broken in this novel quantum condensed state. Although the FFLO state had not been experimentally observed in about 40 years after the theoretical prediction, ${ }^{2,3}$ several evidences for the FFLO state have been obtained in the recent experiments. ${ }^{4}$ The FFLO superconductivity or superfluidity is attracting growing interests not only in the field of superconductivity ${ }^{4}$ but also in the study of cold atom gases ${ }^{5}$ and high density quark matters. ${ }^{6}$

Vast studies of the FFLO state were triggered by the discovery of novel superconducting (SC) phases in the strongly correlated electron systems. ${ }^{4}$ For example, a new SC phase has been identified in the heavy fermion superconductor $\mathrm{CeCoIn}_{5}$ at low temperatures and high magnetic fields. ${ }^{7-16}$ Experimental data for the FFLO state have been obtained in several organic materials, such as $\lambda$-(BETS) ${ }_{2} \mathrm{FeCl}_{4},{ }^{17} \kappa$-(BEDT$\mathrm{TTF})_{2} \mathrm{Cu}(\mathrm{NCS})_{2}{ }^{18,19}$ and (TMTSF) ${ }_{2} \mathrm{ClO}_{4} \cdot{ }^{20,21}$ In these materials the paramagnetic effect which is essential for the FFLO superconductivity is important because the orbital de-pairing effect ${ }^{22}$ is suppressed by the heavy quasiparticle mass and/or the layered crystal structure.

Another common characteristics in these compounds is the presence of the AFQCP in the vicinity of the SC phase. Although the critical spin fluctuation significantly affects the electronic state near the AFQCP, the influence of AFQCP on the FFLO state has not been investigated from the theoretical point of view. The main purpose of this paper is to examine the stability of the FFLO state near the AFQCP. Although the FFLO state has been investigated by Yokoyama et al. ${ }^{23}$ on the basis of the random phase approximation (RPA), the retardation effect, quasiparticle scattering and internal field, which play an essential role in our study, are neglected in the RPA.

We analyze the two-dimensional Hubbard model in order to investigate the FFLO superconductivity near the AFQCP. The model is described as follows,

$$
H=\sum_{\vec{k}, \sigma} \varepsilon(\vec{k}) c_{\vec{k} \sigma}^{\dagger} c_{\vec{k} \sigma}+U \sum_{i} n_{i \uparrow} n_{i \downarrow}-h \sum_{i, \sigma} \sigma n_{i \sigma} .
$$

We consider the square lattice and choose the following tight-binding dispersion,

$$
\varepsilon(\vec{k})=-2 t\left(\cos k_{\mathrm{x}}+\cos k_{\mathrm{y}}\right)+4 t^{\prime} \cos k_{\mathrm{x}} \cos k_{\mathrm{y}}-\mu,
$$

in which the $d$-wave superconductivity is stabilized by the antiferromagnetic (AF) spin fluctuation. ${ }^{24-26}$ In the following, we choose the unit of energy as $2 t=1$ and fix $t^{\prime} / t=0.25$. The chemical potential is chosen so that the number density of electrons is $n=0.9$. The last term in eq. (1) describes the Zeeman splitting where $h=\frac{1}{2} g \mu_{\mathrm{B}} H$ with $H$ being the magnetic field. As for the influence of the magnetic field, we here focus on the Zeeman splitting which is essential for the FFLO superconductivity. $^{2,3}$ We assume a sufficiently large Maki parameter $\alpha=\sqrt{2} H_{\mathrm{c} 2}^{\mathrm{orb}} / H_{\mathrm{c} 2}^{\mathrm{P}}$ and ignore the orbital effect ${ }^{22,27-33}$ for simplicity.

The model eq. (1) is analyzed on the basis of the FLEX approximation $^{34}$ which is based on the Luttinger-Ward formalism. ${ }^{35}$ The critical fluctuation near the AFQCP is taken into account in this approach. ${ }^{25,26}$ The thermodynamic properties, such as the free energy, condensation energy and optical integral have been investigated at zero magnetic field. ${ }^{36}$

It is straightforward to extend the Luttinger-Ward formalism to the FFLO state. We obtain the thermodynamic potential as follows,

$$
\begin{aligned}
& \Omega(T, \mu, h)=\Omega_{0}(T, \mu, h)+\Omega_{\mathrm{F}}+\Omega_{\mathrm{B}}, \\
& \Omega_{\mathrm{F}}=-\sum_{k}\left[\log \left\{\operatorname{det} \tilde{G}(k)^{-1} / \operatorname{det} \tilde{G}^{(0)}(k)^{-1}\right\}\right.
\end{aligned}
$$




$$
\begin{aligned}
& \left.+\sum_{\sigma} G_{\sigma}(k) \Sigma_{\sigma}^{\mathrm{n}}(k)-F(k) \Delta^{\dagger}(k)-F^{\dagger}(k) \Delta(k)\right], \\
& \Omega_{\mathrm{B}}=\left.\Phi\left[G_{\sigma}, F, F^{\dagger}\right]\right|_{\mathrm{st}},
\end{aligned}
$$

where $\Omega_{0}(T, \mu, h)=-T \sum_{\vec{k}, \sigma} \log [1+\exp \{-\beta(\varepsilon(\vec{k})-\sigma h)\}]$ is the thermodynamic potential at $U=0$. We describe the normal and anomalous Green functions as,

$$
\begin{aligned}
\tilde{G}(k) & =\left(\begin{array}{cc}
G_{\uparrow}\left(k_{+}\right) & F(k) \\
F^{\dagger}(k) & -G_{\downarrow}\left(-k_{-}\right)
\end{array}\right), \\
& =\left(\begin{array}{cc}
G_{\uparrow}^{\mathrm{n}}\left(k_{+}\right)^{-1} & \Delta(k) \\
\Delta^{\dagger}(k) & -G_{\downarrow}^{\mathrm{n}}\left(-k_{-}\right)^{-1}
\end{array}\right)^{-1},
\end{aligned}
$$

where $G_{\sigma}^{\mathrm{n}}(k)^{-1}=G_{\sigma}^{(0)}(k)^{-1}-\Sigma_{\sigma}^{\mathrm{n}}(k)$ with $G_{\sigma}^{(0)}(k)=$ $\left(\mathrm{i} \omega_{n}-\varepsilon(\vec{k})+\sigma h\right)^{-1}$. We have described $\vec{k}_{ \pm}=\vec{k} \pm \vec{q}_{\mathrm{F}} / 2$, and $\vec{q}_{\mathrm{F}}$ is the total momentum of Cooper pairs in the FFLO state. For simplicity we assume the Fulde-Ferrel (FF) state where the SC order parameter has the spatial modulation $\Delta(\vec{r})=\exp \left( \pm \mathrm{i} \vec{q}_{\mathrm{F}} \vec{r}\right)$. Since we neglect the Larkin-Ovchinnikov (LO) state, the stability of the FFLO state is underestimated in the following results. Note that the FF state has the same critical magnetic field $H_{\mathrm{c} 2}^{\mathrm{P}}$ as the LO state when the second order phase transition is assumed. We will investigate the LO state in the vicinity of the AFQCP in another publication. ${ }^{37}$

The normal and anomalous self-energies in eq. (7) are obtained from the generating function $\Phi\left[G_{\sigma}, F, F^{\dagger}\right]$ as,

$$
\begin{aligned}
& \Sigma_{\sigma}^{\mathrm{n}}(k)=\frac{\delta \Phi}{\delta G_{\sigma}(k)}, \\
& \Delta(k)=-\frac{\delta \Phi}{\delta F^{\dagger}(k)}, \\
& \Delta^{\dagger}(k)=-\frac{\delta \Phi}{\delta F(k)},
\end{aligned}
$$

by which the stationary conditions are satisfied as, ${ }^{35,36}$

$$
\frac{\delta \Omega}{\delta \Sigma_{\sigma}^{\mathrm{n}}(k)}=\frac{\delta \Omega}{\delta \Delta(k)}=\frac{\delta \Omega}{\delta \Delta^{\dagger}(k)}=0
$$

In the FLEX approximation the generating function is given as,

$$
\begin{aligned}
& \Phi\left[G_{\sigma}, F, F^{\dagger}\right]=U n_{\uparrow} n_{\downarrow}+\sum_{q}\left\{\log \left[1-U \chi_{ \pm}^{0}(q)\right]\right. \\
& +\frac{1}{2} \log \left[\left(1-U \chi_{\mathrm{F}}^{0}(q)\right)^{2}-U \chi_{\uparrow}^{0}(q) \chi_{\downarrow}^{0}(q)\right] \\
& \left.+\frac{1}{2} U^{2}\left[\chi_{\mathrm{F}}^{0}(q)^{2}+\chi_{\uparrow}^{0}(q) \chi_{\downarrow}^{0}(q)\right]+U\left[\chi_{ \pm}^{0}(q)+\chi_{\mathrm{F}}^{0}(q)\right]\right\},
\end{aligned}
$$

where the irreducible susceptibilities are described as,

$$
\begin{aligned}
& \chi_{ \pm}^{0}(q)=-\sum_{k}\left[G_{\uparrow}(k+q) G_{\downarrow}(k)+F(k+q) F^{\dagger}(-k)\right] \\
& \chi_{\sigma}^{0}(q)=-\sum_{k} G_{\sigma}(k+q) G_{\sigma}(k), \\
& \chi_{\mathrm{F}}^{0}(q)=-\sum_{k} F^{\dagger}(k+q) F(k) .
\end{aligned}
$$

We have used the notations $\sum_{k}=T / N \sum_{\omega_{n}, \vec{k}}$ and $\sum_{q}=$
$T / N \sum_{\Omega_{n}, \vec{q}}$ where $\omega_{n}=(2 n+1) \pi T, \Omega_{n}=2 n \pi T, T$ is the temperature and $N$ is the number of sites. The unit $\hbar=c=k_{\mathrm{B}}=1$ is used throughout this paper.

We carry out the numerical calculation on the $N=$ $64 \times 64$ sites and takes the Matsubara frequency $N_{\mathrm{f}}=$ 2048 for $T \geq 0.004$. We keep the cutoff of Matsubara frequency as $\left(N_{\mathrm{f}}+1\right) \pi T>12.8$ for $T<0.004$, which is larger than the band width $W=4$.

In the following results, the dominant component of

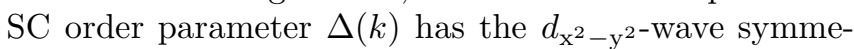
try. The order parameter has an admixed odd frequency

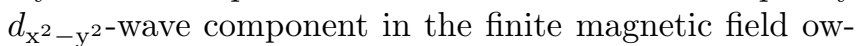
ing to the violation of time reversal symmetry. The even frequency $p$-wave component is also admixed with the $d_{\mathrm{x}^{2}-\mathrm{y}^{2}}$-wave component in the FFLO state due to the breakdown of inversion symmetry like in the noncentrosymmetric superconductors. ${ }^{38,39}$ The extended $s$ wave component is also induced in the FFLO state unless $\vec{q}_{\mathrm{F}} \|[110]$. We will show that the $p$-wave order parameter plays an quantitatively important role for the stability of the FFLO state, while the other admixed components are negligible. In the following we assume the FFLO modulation vector $\vec{q}_{\mathrm{F}}$ along the [110]-axis for the numerical accuracy, although a slightly larger condensation energy is obtained for $\vec{q}_{\mathrm{F}} \|[100]$. We have confirmed that qualitatively the same results are obtained for $\vec{q}_{\mathrm{F}} \|$ [100]. The direction of the modulation vector $\vec{q}_{\mathrm{F}}$ is actually affected by the orbital effect ${ }^{30,31}$ which is neglected in this paper.

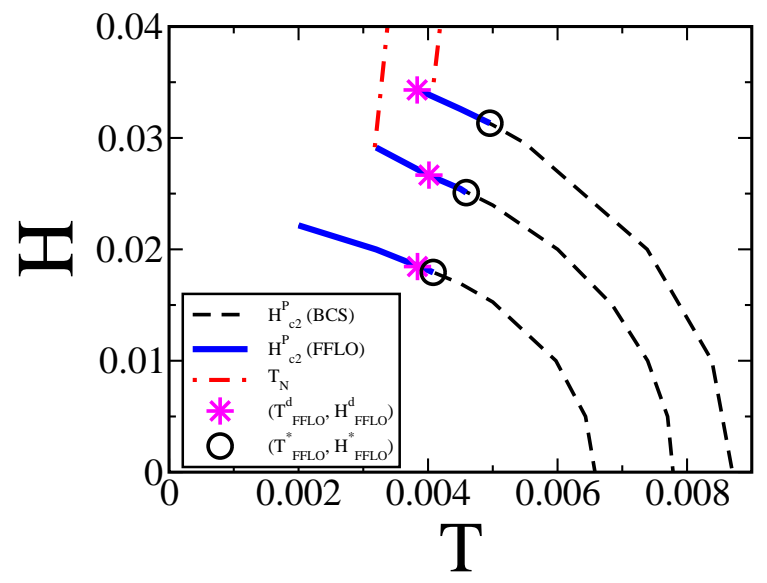

Fig. 1. (Color online) Paramagnetic critical magnetic field $H_{\mathrm{c} 2}^{\mathrm{P}}$ for $U / t=2.8,3.1$ and 3.4 from the bottom to the top. The solid and dashed lines show the $H_{\mathrm{c} 2}^{\mathrm{P}}$ for the FFLO and BCS state, respectively. The tricritical point $\left(T_{\mathrm{FFLO}}^{*}, H_{\mathrm{FFLO}}^{*}\right)$ is shown by the circles $(\bigcirc)$. The stars show the tricritical point $\left(T_{\mathrm{FFLO}}^{\mathrm{d}}, H_{\mathrm{FFLO}}^{\mathrm{d}}\right)$ obtained by neglecting the $\vec{q}_{\mathrm{F}}$ dependence of the order parameter $\Delta(k)$. The Neel temperature $T_{\mathrm{N}}$ is shown by the dash-dotted line for $U / t=3.1$ and 3.4.

First we investigate the tricritical point $\left(T_{\mathrm{FFLO}}^{*}, H_{\mathrm{FFLO}}^{*}\right)$ where the transition line from the FFLO state to the uniform BCS state merges to the critical magnetic field $H_{\mathrm{c} 2}^{\mathrm{P}}(T)$. Figure 1 shows the results of $H_{\mathrm{c} 2}^{\mathrm{P}}(T)$ and $\left(T_{\mathrm{FFLO}}^{*}, H_{\mathrm{FFLO}}^{*}\right)$ for $U / t=2.8,3.1$ and 3.4. We show the transition temperature $T_{\mathrm{N}}$ from the normal state to the $\mathrm{AF}$ state in the same figure. ${ }^{40}$ 
The Neel temperature $T_{\mathrm{N}}$ is determined by using the criterion $1-U \chi_{ \pm}^{0}(q)=0.005$ at $\vec{q}=(\pi, \pi)$ and $\Omega_{\mathrm{n}}=0$, where the AF order occurs with the magnetic moment perpendicular to the magnetic field. We find that the paramagnetic limit of the critical magnetic field $H_{\mathrm{c} 2}^{\mathrm{P}}$ is significantly enhanced by the internal field arising from the AF spin fluctuation. This is consistent with the experimental results in $\mathrm{CeCoIn}_{5} .{ }^{15}$

We see that the FFLO state is stable in the vicinity of the AFQCP. The temperature at the tricritical point $T_{\text {FFLO }}^{*}$ in Fig. 1 (see also Fig. 2) is larger than that obtained in the weak coupling BCS theory $T_{\mathrm{FFLO}}^{*}=$ $0.56 T_{\mathrm{c} 0}{ }^{4,41}$ where $T_{\mathrm{c} 0}$ is the critical temperature at zero magnetic field. This result seems to be surprising because the FFLO state is destabilized by the finite quasiparticle lifetime $\tau^{*}(\vec{k})^{30,31,41}$ which is significantly decreased by the critical spin fluctuation. We here obtain the scattering rate $\Gamma_{\sigma}(\vec{k})=1 / \tau_{\sigma}^{*}(\vec{k})=-z_{\sigma}(\vec{k}) \operatorname{Im} \Sigma_{\sigma}^{\mathrm{R}}(\vec{k}, 0) \sim 2.3 T_{\mathrm{c} 0}$ at $\vec{k}=(\pi, 0)$ and $\sigma=\uparrow$ for $U / t=3.1$ and $(T, H)=$ $\left(T_{\mathrm{FFLO}}^{*}, H_{\mathrm{FFLO}}^{*}\right)$. Here, $z_{\sigma}(\vec{k})=\left(1-\partial \operatorname{Re} \Sigma_{\sigma}^{\mathrm{R}}(\vec{k}, \omega) / \partial \omega\right)^{-1}$ is the mass renormalization factor. This value of $\Gamma(\vec{k})$ is larger than the critical value $\Gamma \sim 1.4 T_{\mathrm{c} 0}$ where the FFLO state disappears owing to the impurity scattering.

The influence of the short lifetime is overcame by the other strong coupling effects, such as the retardation effect and the parity mixing. We have confirmed that the frequency dependence of the SC order parameter $\Delta(k)$ significantly increases the ratio $T_{\mathrm{FFLO}}^{*} / T_{\mathrm{c} 0}$. The parity

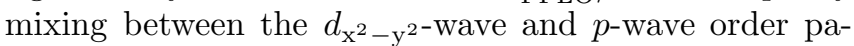
rameters furthermore enhances the FFLO superconductivity as shown in Fig. 1. The stars in Fig. 1 show the tricritical point $\left(T_{\mathrm{FFLO}}^{\mathrm{d}}, H_{\mathrm{FFLO}}^{\mathrm{d}}\right)$ where the momentum and frequency dependences of the order parameter $\Delta(k)$ is fixed to be those in the uniform BCS state. We see that the FFLO state is stabilized by the parity mixing, particularly near the AF critical point. Thus, the FFLO superconductivity is stabilized by the strong coupling effects near the AFQCP. This is consistent with the fact that the experimental indications for the FFLO superconductivity have been obtained in the strongly correlated electron systems near the AFQCP. ${ }^{4,7,8,17-21}$

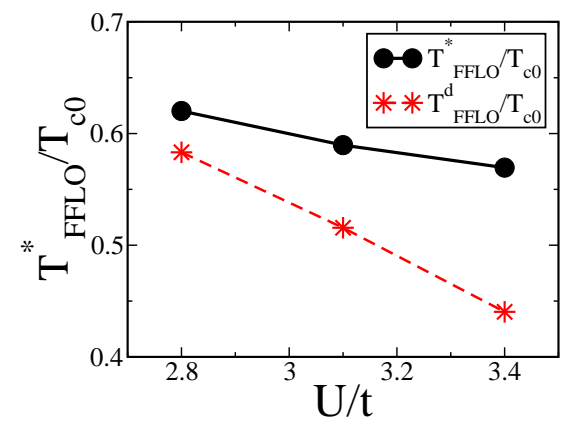

Fig. 2. (Color online) $U$-dependence of the ratio $T_{\mathrm{FFLO}}^{*} / T_{\mathrm{c} 0}$ which represents the stability of the FFLO state. We show the $T_{\mathrm{FFLO}}^{\mathrm{d}} / T_{\mathrm{c} 0}$ for a comparison.

Next, we discuss the $U$-dependence of the FFLO instability. While the closeness to the AFQCP can be ex- perimentally controlled by the pressure or carrier doping, that is represented by the parameter $U$ in our calculation. Figure 2 shows the ratio $T_{\mathrm{FFLO}}^{*} / T_{\mathrm{c} 0}$ which decreases as increasing $U$ and approaching to the AF instability. This is mainly because of the quasiparticle scattering which is more sensitive to the closeness to the AFQCP than the other strong coupling effects. Thus, the FFLO state becomes unstable little by little as approaching to the AFQCP. This result is consistent with the pressure dependence of $\mathrm{CeCoIn}_{5},{ }^{15}$ where $T_{\mathrm{FFLO}}^{*} / T_{\mathrm{c} 0}$ gradually increases as increasing the pressure.

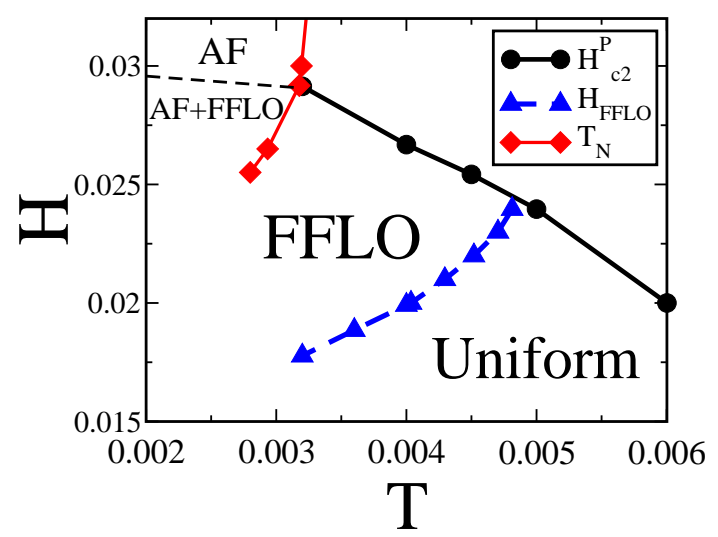

Fig. 3. (Color online) $H-T$ phase diagram for $U / t=3.1$ at high magnetic fields and low temperatures. Triangles show the second order phase transition from the uniform BCS state to the FFLO state. Diamonds show the second order phase transition to the AF state. We described the schematic thin dashed line below which the FFLO superconductivity coexists with the AF order.

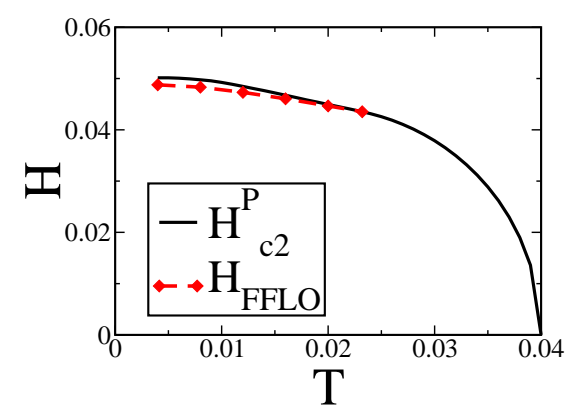

Fig. 4. (Color online) $H-T$ phase diagram of the BCS model eq. (16). The solid line shows the $H_{\mathrm{c} 2}^{\mathrm{P}}$. The FFLO state is stabilized in the narrow region between the solid and dashed lines.

Finally, we investigate the phase transition from the uniform BCS state to the FFLO state. Figure 3 shows the $H-T$ phase diagram for $U / t=3.1$. It is shown that the critical magnetic field $H_{\mathrm{FFLO}}(T)$ from the uniform BCS state to the FFLO state rapidly decreases as decreasing the temperature. The positive value of the slope $\mathrm{d} H_{\mathrm{FFLO}} / \mathrm{d} T$ is much larger than that in the weak coupling BCS theory. ${ }^{42}$ This is because the SC gap leads to the spin gap in the magnetic excitation and decreases the quasiparticle scattering rate. FFLO superconductivity is 
enhanced below $T_{\mathrm{c}}$ since the lifetime of quasiparticles significantly increases. We obtain the convex curvature $\left(\mathrm{d}^{2} H_{\mathrm{FFLO}} / \mathrm{d} T^{2}>0\right)$ which is consistent with the experiments in $\mathrm{CeCoIn}_{5} \cdot{ }^{4,15}$ The slope $\mathrm{d} H_{\mathrm{FFLO}} / \mathrm{d} T$ decreases as decreasing the temperature because the quasiparticle scattering rate almost vanishes at low temperatures in the $\mathrm{SC}$ state. We note that the concave curvature $\mathrm{d}^{2} H_{\mathrm{FFLO}} / \mathrm{d} T^{2}<0$ is obtained in Ref. 31 where the orbital effect is taken into account but the influences of the spin fluctuation are neglected. ${ }^{43}$ Thus, the characteristics of the phase transition from the BCS state to the FFLO state in $\mathrm{CeCoIn}_{5}$ are reproduced by taking into account the critical spin fluctuation, while they seem to be incompatible with the weak coupling BCS theories. ${ }^{4,22,31,42}$

To illuminate the influence of the spin fluctuation more clearly we show the H-T phase diagram in the BCS theory. We obtain Fig. 4 by solving the following model,

$$
H=\sum_{\vec{k}, \sigma} \varepsilon(\vec{k}) c_{\vec{k} \sigma}^{\dagger} c_{\vec{k} \sigma}+g_{\mathrm{s}} \sum_{\vec{k}, \overrightarrow{k^{\prime}}} B^{\dagger}\left(\overrightarrow{k^{\prime}}\right) B(\vec{k})-h \sum_{i, \sigma} \sigma n_{i \sigma}
$$

on the basis of the BCS mean field theory. Here, $B(\vec{k})=$ $\phi_{\mathrm{d}}(\vec{k}) c_{\vec{k}_{+}, \uparrow} c_{-\vec{k}_{-} \downarrow}$ and $\phi_{\mathrm{d}}(\vec{k})=\cos k_{\mathrm{x}}-\cos k_{\mathrm{y}}$. This model has been used in the weak coupling theories for the $d$ wave FFLO state. ${ }^{31,41}$ We adopt the dispersion relation $\varepsilon(\vec{k})$ as eq. (2) and choose the attractive interaction $g_{\mathrm{s}}<$ 0 so that $T_{\mathrm{c} 0}=0.04$. We assume the FF state and $\vec{q}_{\mathrm{F}} \|$ [110] to compare Fig. 4 with Fig. 3 on an equal footing. We see that the FFLO state is stable in the narrow region just below $T_{\mathrm{c}}$ in Fig. 4. Thus, the FFLO state is much more stable in the repulsive Hubbard model (Fig. 3) than the BCS model (Fig. 4). This comparison illuminates the enhancement of the FFLO state near the AFQCP.

The phase diagram in Fig. 3 seems to be consistent with the experimental results in $\mathrm{CeCoIn}_{5}$, but there remains a discrepancy between our results and experiments. Although the first order phase transition from the normal to $\mathrm{SC}$ state occurs in $\mathrm{CeCoIn}_{5}$ above $T=$ $T_{\text {FFLO }}^{*}{ }^{44,45}$ the SC transition is in the second order in our calculation for $T>0.0032$. This is because the quasiparticle scattering ${ }^{41}$ as well as the internal magnetic field ${ }^{46}$ arising from the AF spin fluctuation suppress the first order phase transition. Therefore, the first order phase transition in $\mathrm{CeCoIn}_{5}$ above $T=T_{\mathrm{FFLO}}^{*}$ should be attributed to the other origin rather than the AFQCP. For example, the orbital effect neglected in our calculation induces the first order phase transition above $T_{\text {FFLO }}^{*} \cdot{ }^{30,31}$ Furthermore, we found that the unequal effective mass between two spin species in $\mathrm{CeCoIn}_{5}{ }^{47,48}$ also induces the first order phase transition, as will be shown in another publication. ${ }^{37}$ The heavy fermion physics, such as the strong electron correlation and the hybridyzation of localized and conduction electrons, may also affect the order of phase transition.

At the last of this paper we comment on the magnetic instability in the FFLO state. Figure. 3 shows the phase transition from the FFLO state to the AF state (Diamonds) in contrast to the result at the zero magnetic field where the AF spin correlation decreases as decreas- ing the temperature below $T_{\mathrm{c}}$. Our result in Fig. 3 shows that the AF order can coexist with the FFLO state due to the large residual density of states in the FFLO state. We found that the AF order is furthermore enhanced in the LO state where the Andreev bound state at the spatial line node of SC order parameter induces the magnetic moment. ${ }^{37}$ These results may be consistent with the NMR measurement in $\mathrm{CeCoIn}_{5},{ }^{49}$ although the discrepancy seems to remain in the experimental data. ${ }^{11,13,49}$

In conclusion, we found that the FFLO superconductivity is stable near the AFQCP even though the quasiparticle scattering rate is significantly increased by the critical spin fluctuation. The quasiparticle scattering is destructive to the FFLO state, but almost canceled by the strong coupling effect. The FFLO state is slightly suppressed as approaching to the AFQCP mainly because the quasiparticle scattering is increased. The phase diagram obtained in our calculation is consistent with the experimental results in $\mathrm{CeCoIn}_{5}$, however the first order phase transition is not reproduced. We pointed out some possible mechanisms for the first order phase transition in $\mathrm{CeCoIn}_{5}$. The possible magnetic instability in the FFLO state was discussed.

The authors are grateful to R. Ikeda, K. Izawa, Y. Matsuda for fruitful discussions. Numerical computation in this work was carried out at the Yukawa Institute Computer Facility.

1) J. Bardeen, L. N. Cooper and J. R. Schrieffer: Phys. Rev. 108 (1957) 1175.

2) P. Fulde and R. A. Ferrel: Phys. Rev. 135 (1964) A550.

3) A. I. Larkin and Yu. N. Ovchinnikov: Zh. Eksp. Teor. Fiz. 47 (1964) 1136 [Sov. Phys. JETP 20 (1965) 762.]

4) Y. Matsuda and H. Shimahara: J. Phys. Soc. Jpn. 76 (2007) 051005 and references there in.

5) M. W. Zwierlein et al.: Science 311 (2006) 492; G. B. Partridge et al.: Science 311 (2006) 503.

6) R. Casalbuoni and G. Nardulli: Rev. Mod. Phys. 76 (2004) 263.

7) H. A. Radovan et al.: Nature 425 (2003) 51.

8) A. Bianchi et al.: Phys. Rev. Lett. 91 (2003) 187004.

9) T. Watanabe et al.: Phys. Rev. B 70 (2004) 020506(R).

10) C. Capan et al.: Phys. Rev. B 70 (2004) 134513.

11) K. Kakuyanagi et al.: Phys. Rev. Lett. 94 (2005) 047602.

12) C. Martin et al.: Phys. Rev. B 71 (2005) 020503.

13) V. F. Mitrovic et al.: Phys. Rev. Lett. 97 (2006) 117002.

14) K. Kumagai et al.: Phys. Rev. Lett. 97 (2006) 227002.

15) C. F. Miclea et al.: Phys. Rev. Lett. 96 (2006) 117001.

16) V. F. Correa et al.: Phys. Rev. Lett. 98 (2007) 087001

17) S. Uji et al.: Phys. Rev. Lett. 97 (2006) 157001.

18) J. Singleton et al.: J. Phys. Condens. Matter 12 (2000) L641.

19) R. Lortz et al.: Phys. Rev. Lett. 99 (2007) 187002.

20) J. Shinagawa et al.: Phys. Rev. Lett. 98 (2007) 147002.

21) S. Yonezawa et al.: arXiv:0801.0484; arXiv:0804.1524.

22) L. W. Gruenberg and L. Gunther: Phys. Rev. Lett. 16 (1966) 996.

23) T. Yokoyama et al.: arXiv:0706.3270.

24) D. J. Scalapino, Phys. Rep. 250 (1995) 329.

25) T. Moriya and K. Ueda, Adv. Phys. 49 (2000) 555.

26) Y. Yanase et al.: Phys. Rep. 387 (2004) 1.

27) M. Tachiki et al.: Z. Phys. B 100 (1996) 369.

28) H. Shimahara and D. Rainer: J. Phys. Soc. Jpn. 66 (1997) 3591.

29) M. Houzet and A. Buzdin: Phys. Rev. B 63 (2001) 184521.

30) H. Adachi and R. Ikeda: Phys. Rev. B 68 (2003) 184510.

31) R. Ikeda: Phys. Rev. B 76 (2007) 134504; 054517.

32) T. Maniv, V. Zhuravlev: arXiv:0712.3981v1.

33) T. Mizushima et al.: Phys. Rev. Lett. 95 (2005) 117003; M. 
Ichioka et al.: Phys. Rev. B 76 (2007) 014503.

34) N. E. Bickers et al.: Phys. Rev. Lett. 62 (1989) 961;

35) J. M. Luttinger and J. C. Ward, Phys. Rev. 118 (1960) 1417.

36) Y. Yanase and M. Ogata: J. Phys. Soc. Jpn. 74 (2005) 1534.

37) Y. Yanase: unpublished.

38) V. M. Edelstein: Sov. Phys. JETP 68 (1989) 1244; P. A. Frigeri et al.: Phys. Rev. Lett 92 (2004) 097001; S. Fujimoto: J. Phys. Soc. Jpn. 76 (2007) 051008; Y. Yanase and M. Sigrist: J. Phys. Soc. Jpn. 76 (2007) 043712.

39) The parity mixing in the FFLO state has been pointed out in S. Matsuo et al.: J. Phys. Soc. Jpn. 63 (1994) 2499.

40) The field induced AF order has been investigated in K. Sakurazawa et al.: J. Phys. Soc. Jpn. 74 (2005) 271.

41) D. F. Agterberg and K. Yang: J. Phys. Condens. Matter 13
(2001) 9259.

42) A. B. Vorontsov et al.: Phys. Rev. B 72 (2005) 184501.

43) The large positive slope and the convex curvature of $H_{\mathrm{FFLO}}(T)$ have been obtained in Ref. 30 , but the quartic correction which is important for $H_{\mathrm{FFLO}}(T)$ has been neglected. See Refs. 31 and 32 .

44) A. Bianchi et al.: Phys. Rev. Lett. 89 (2002) 137002.

45) T. Tayama et al.: Phys. Rev. B 65 (2002) 180504.

46) A. B. Vorontsov and M. J. Graf: Phys. Rev. B 74 (2006) 172504.

47) R. Settai et al.: J. Phys.: Condens. Matter 13 (2001) L627; A. McCollam et al.: Phys. Rev. Lett. 94 (2005) 186401.

48) S. Onari et al.: J. Phys. Soc. Jpn. 77 (2008) 023703.

49) B.-L. Young et al.: Phys. Rev. Lett. 98 (2007) 036402. 